\section{PRECISION SHADE MATCHING}

Universal composite Venus features superior handling properties, precision shade matching and blending with the exclusive Color Adaptive Matrix system and offers a range of 27 shades.

In addition to the standard shades, which are adjusted to match the Vita shade guide, you also have at your disposal custom shades and very light shades (Super Bleach) for treating bleached teeth.
A 2Layer shade guide using genuine materials makes it exceptionally easy to select the most appropriate shade. Owing to the Colour Adaptive Matrix, fillings using Venus blend in perfectly with the dental hard tissue surrounding them. Venus also features optimum physical properties, is extremely easy to work and highly abrasion-resistant.

Reader response number 58

sensitive teeth. Savodent is quick and easy to use - two active foam strips are placed in a double tray, which is then worn for just ten minutes per treatment, giving immediate relief from the pain of sensitive teeth.

One single application gives relief for up to one month. The active ingredient, potassium oxalate, has been shown to be the best formula for occluding exposed dentinal tubules and reducing pain by modifying the nerve excitability. Savodent provides significantly faster and longer lasting results than sensitive teeth toothpastes.

Reader response number 59

\section{RANGE THAT REPAIRS ENAMEL}

BioRepair offers an effective solution against acid attacks and erosion and can repair enamel due to its patented scientific formula. BioRepair incorporates a component historically used in bone surgery and contains Microrepair microparticles made of carbonate-hydroxyapatite - the same chemical substance that makes up tooth enamel and dentine. These microparticles effectively fill in any microscopic cracks or holes, thereby repairing the damaged enamel surface.

All products in the BioRepair range are suitable for the whole family to use. They help prevent tooth decay and do not contain any added fluoride - the carbonate-hydroxyapatite within BioRepair fulfils a similar function to fluoride but can be used safely in higher concentrations to give faster results.
BioRepair Plus Total Protection toothpaste repairs daily wear before it can lead to decay, now with 33\% more Microrepair than before. It provides daily protection against acid erosion, plaque and tartar, while also helping to keep breath fresh.

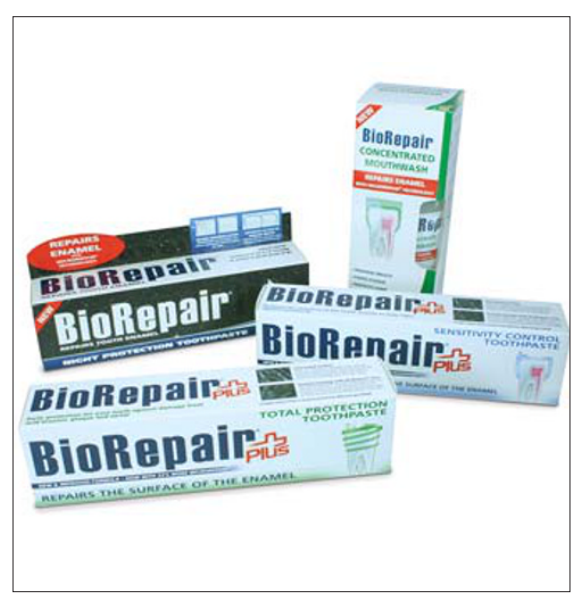

\section{IMPLANT SURFACE TECHNOLOGY}

Straumann's SLActive implant surface technology features chemical purity and retained surface energy that shows notable hydrophilic (fluid attracting) properties, allowing direct cell reaction to take place at the initial stage of the

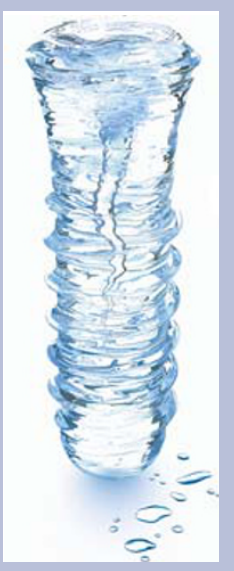
osseointegration process.

Bone formation is initiated at an earlier stage, providing higher implant stability and reducing the risk of implant failure, desirable for those patients with high risk factors and who suffer from poor bone quality.

In addition, Straumann's new Roxolid implant ensures both clinicians and patients benefit from high treatment security and increased predictability in even the most challenging of procedures.

Reader response number 60
The range also includes BioRepair Plus Sensitivity Control toothpaste. Its bioactive microparticles bond with the natural dentine and enamel to form a protective layer over nerve endings within the tooth, relieving excessive pain caused by brushing and by hot or cold food and drink.

BioRepair Night Protection Toothpaste helps to guard the teeth from acid attacks during the night. It also contains zinc, which offers increased antiseptic and anti-halitosis action. Lastly, BioRepair Concentrated Mouthwash is an enamel repair mouthwash which protects the gums, freshens breath and effectively combats the build up of plaque and tartar by reaching those difficult areas, allowing the antiseptic action to really get to work on the whole mouth.

Reader response number 61 